\title{
NETWORK STRUCTURES OF SYMPTOMS FROM THE ZUNG DEPRESSION SCALE
}

\author{
Giovanni Briganti ${ }^{* 1}$, Marco Scutari $^{2}$, and Paul Linkowski ${ }^{1}$ \\ ${ }^{1}$ Unit of Epidemiology, Biostatistics, and Clinical Research, Université libre de Bruxelles \\ ${ }^{2}$ IDSIA Dalle Molle Institute for Artificial Intelligence
}

June 23, 2020

This paper was peer-reviewed and accepted for publication in Psychological Reports.

\begin{abstract}
The Self-rating Depression Scale (SDS) is a psychometric tool composed of 20 items used to assess depression symptoms. The aim of this work is to perform a network analysis of this scale in a large sample composed of 1090 French-speaking Belgian university students. We estimated a regularized partial correlation network and a Directed Acyclic Graph for the 20 items of the questionnaire. Node predictability (shared variance with surrounding nodes in the network) was used to assess the connectivity of items. The network comparison test was performed to compare networks from female and male students. The network composed of items from the SDS is overall positively connected, although node connectivity varies. Item 11 ("My mind is as clear as it used to be") is the most interconnected item. Networks from female and male students did not differ. DAG reported directed edges among items. Network analysis is a useful tool to explore depression symptoms and offers new insight as to how they interact. Further studies may endeavor to replicate our findings in different samples, including clinical samples to replicate the network structures and determine possible viable targets for clinical intervention.
\end{abstract}

network analysis; directed acyclic graphs; students

\section{Introduction}

Depression (also known as Major Depressive Disorder, MDD) is a common psychiatric disorder (Goldberg, 2011), and the top cause of disability worldwide (Lopez et al., 2006). In the fifth edition of the Diagnostic and Statistical Manual of Mental Disorders or DSM (American Psychiatric Association, 2013), depression is characterized as a combination of five symptoms in a list of nine, including depressed mood or anhedonia (this symptom must be included in each combination), increase or decrease in weight or appetite, fatigue, sleep problems and suicidal ideation. Depression is then defined as a heterogenous disorder, which leads to an important symptom variability (Fried and Nesse, 2015).

In most depression scales, such as the Hamilton Rating Scale for Depression (HRSD), sum scores are used to explain the severity of the disorder (Hamilton, 1960). Zung created in 1965 a self-rating depression scale (SDS) using 20 items representing symptoms from three conceptual domains (Zung, 1965): pervasive effects (e.g. item 1: "I feel downhearted and blue"), physiological equivalents (e.g. item 4: I have trouble sleeping at night") and psychological equivalents (e.g. item 19: "I feel that others would be better off if I were dead"). In both these approaches, symptoms are considered as interchangeable measures of depression.

In recent years, the network approach to psychopathology has been proposed as a novel way of analyzing mental disorders as complex systems (Borsboom and Cramer, 2013). This approach considers that a mental disorder arises from connections among its symptoms, that can cause each other (Borsboom, 2017): this conceptualization differs from the latent variable theory, which states that symptoms are passive consequences

\footnotetext{
${ }^{*}$ Corresponding author. Email: giovanni.briganti@hotmail.com
} 
of an underlying cause. In psychiatry, the latter is unlikely to identify a specific mechanism that causes a mental disease (Borsboom, 2008), which makes the network approach an interesting alternative. Five principles of network theory proposed by Borsboom are likely to apply in the case of depression: complexity (mental disorders are characterized as interactions between the components of a network, such as symptoms), symptom-component correspondence (components in a network are problems defined as symptoms), direct causal connections (symptoms cause each other in a network), network structure of mental disorders (certain symptoms are more connected than others and symptoms from the same network often arise together) and hysteresis (symptoms activate each other even after the trigger cause has disappeared). Hysteresis has been proven to be a dynamic present in depression (Cramer et al., 2016): a dormant (asymptomatic) network of depression is present in the healthy individual: a trigger cause may activate one or more symptoms which, in turn, activate the rest of the network in a "pathological" condition. Mental health can then be defined as a stable state of a weakly connected network (Borsboom, 2017).

Network analysis is a set of statistical techniques developed in the conceptual framework of network theory to identify network structures in datasets. Network analysis has been used in various fields, such as empathy (Briganti et al., 2018), posttraumatic stress disorder (Fried et al., 2018), schizophrenia (Galderisi et al., 2018) and self-worth (Briganti et al., 2019).

Depression constitutes a domain of interest in network analysis (Mullarkey et al., 2018): the network literature has counted several studies in this domain in the last few years on both the cross-sectional and the dynamic level (Fried et al., 2017; Fried and Haslbeck, 2019; St Quinton and Stain, 2019; McNally et al., 2017; Wichers, 2014). Links between depressive symptoms and inflammation markers have been explored (Fried and Haslbeck, 2019), as well as comorbidity with obsessive-compulsive disorder (McNally et al., 2017). However, the symptoms from SDS have never been studied as a network structure: it is particularly interesting to do so because the SDS is a self-reported measure (as opposed to the HRSD or other clinimetric indices), and although network structures have been reported to be stable across different samples (Fried et al., 2018), the similarity of network connections between similar symptoms across different scales has yet to be discussed.

Researchers usually analyze constructs as undirected networks composed of nodes (symptoms) and undirected connections representing regularized partial correlations (Epskamp and Fried, 2018). Studying connections ("edges") as pairwise relationships between symptoms is important when considering mental illness as a complex system: if two symptoms $\mathrm{X}$ and $\mathrm{Y}$ are connected in the network, then $\mathrm{X}$ predicts $\mathrm{Y}$, and vice versa (Briganti et al., 2018). Based on the connectivity of network components, which is often measured as the shared variance of a given node with surrounding nodes (this measure is often referred to as node predictability), one can identify symptoms that greatly contribute to the self-determination of the network and could therefore serve as meaningful targets for clinical intervention (Haslbeck and Fried, 2017); in the case of the SDS, it is therefore interesting to find which items are important in the network structure.

However, in the case of depression, it is also interesting to identify a causal pathway might help to gather information about the sequence of symptom activation in a previously healthy individual. Such information cannot be gathered while studying undirected network structures, and other statistical models are to be applied. Causal pathways can for instance be identified in Bayesian networks and more precisely with Directed Acyclic Graphs (DAGs), which are probabilistic models. DAGs are capable of learning the underlying causal graphs from the data (Moffa et al., 2017), and are therefore represented as networks with directed connections; they are well established in the network literature (Scutari and Denis, 2015). Because they can provide a network structure with directed connections among nodes, DAGs can be complementary to the more popular partial correlation networks in the field of psychopathological networks, and have been recently used to explore depression (McNally et al., 2017). Studying the network structures of common psychometric scales such as the SDS is important, since discovering connections among symptoms in different kinds of samples helps towards building better computational models (Robinaugh et al., 2019).

Our work therefore aims to build on previous papers and apply network modeling to depression symptoms as described in the SDS using both directed and undirected network models. This paper will be exclusively focused on the study of the construct of depression as presented by the SDS with both partial correlation networks and DAGs. First, we will estimate a regularized partial correlation network composed of symptoms from the SDS and explore its overall connectivity. Second, we will explore the connectedness of items from the SDS with well-established centrality measures (Briganti et al., 2018). Third, we will explore causal pathways between SDS symptoms using DAG constraint-based structure learning algorithms. 


\section{Method}

\subsection{Participants}

This study is carried out on a dataset composed of 1090 French-speaking Belgian university students ( $54 \%$ females, $46 \%$ males), aged 17 to 25 years old $(\mathrm{M}=19.52 ; \mathrm{SD}=1.67)$.

\subsection{Measurement}

The Self-Rating Depression Scale (SDS) is composed of 20 items (Table 1) meant to assess depression symptoms. Item scores range from 1 ("a little of the time) to 4 ("most of the time"). Items are presented in their original order in the questionnaire. Certain items are reverse scored (items 2, 5, 6, 11, 12, 14, 16, 17, 18 and 20), such as item 6 ("I still enjoy sex").

Table 1: The Self-Rating Depression Scale (Zung, 1965)

\begin{tabular}{|l|l|}
\hline $\mathrm{N}$ & Item \\
\hline 1 & I feel down hearted and blue \\
\hline 2 & Morning is when I feel the best \\
\hline 3 & I have crying spells or feel like it \\
\hline 4 & I have trouble sleeping at night \\
\hline 5 & I eat as much as I used to \\
\hline 6 & I still enjoy sex \\
\hline 7 & I notice that I am losing weight \\
\hline 8 & I have trouble with constipation \\
\hline 9 & My heart beats faster than usual \\
\hline 10 & I get tired for no reason \\
\hline 11 & My mind is as clear as it used to be \\
\hline 12 & I find it easy to do the things that I used to \\
\hline 13 & I am restless and cannot keep still \\
\hline 14 & I feel hopeful about the future \\
\hline 15 & I am more irritable than usual \\
\hline 16 & I find it easy to make decisions \\
\hline 17 & I feel that I am useful and needed \\
\hline 18 & My life is pretty full \\
\hline 19 & I feel that others would be better off if I were dead \\
\hline 20 & I still enjoy the things I used to \\
\hline
\end{tabular}

\subsection{Network analysis}

Network analysis is carried out in R (open source, available at https://www.r-project.org/). Packages used to carry out the analysis include qgraph (Epskamp et al., 2012) and glasso (Friedman et al., 2014) for network estimation and visualization, mgm for node predictability (Haslbeck and Waldorp, 2016), bootnet (Epskamp and Fried, 2018) for stability, NetworkComparisonTest (van Borkulo et al., 2014), bnlearn (Scutari, 2010) and pcalg (Kalisch et al., 2012) for the estimation of directed acyclic graphs.

\subsubsection{Regularized partial correlation network}

Network estimation A network is composed of nodes (representing items from the SDS) and edges (connections between items). A correlation matrix is used as input to estimate a Gaussian Graphical Model (GGM), which is a regularized partial correlation network. Each edge has a weight (parameter resulting from the GGM) which is regularized using a graphical lasso (least absolute shrinkage and selection operator) to avoid the estimation of spurious edges and therefore provides a conservative model (Epskamp and Fried, 2018). An edge therefore represents a regularized partial correlation between two symptoms, controlling for 
all other symptoms in the network. The thickness and color saturation of an edge represent its weight (the strength of the association between two nodes); edges can therefore be positive (blue) or negative (red). In the case of the SDS, a self-report scale which measures depression symptoms, a positive edge can be statistically interpreted as following: if two given nodes $\mathrm{X}$ and $\mathrm{Y}$ share an edge $\mathrm{XY}$ in the network, and the observed group of subjects scores high on $\mathrm{X}$, then the observed group is also more likely to score high on $\mathrm{Y}$ (Briganti et al., 2018). On the other hand, a negative edge implies that if an observed group of subjects scores high on X, then the observed group is less likely to score high on Y. Two nodes will be disconnected if they are conditionally independent. Nodes are placed in the network by the Fruchterman-Reingold algorithm, based on the sum of connections a given node has with other nodes (Fruchterman and Reingold, 1991).

Network inference Node predictability is an absolute measure of the interconnectedness of a node (Haslbeck and Waldorp, 2016) and is the percentage of variance of a given node explained by surrounding nodes. Node predictability has been described as the upper bound of controllability: "if one assumes that all edges for node A are directed towards that node, predictability provides an estimate of how much influence we can have on A via all other nodes" (Briganti et al., 2019). Node predictability is represented in the network as a pie chart surrounding the node.

Network Comparison Test We performed a Network Comparison Test (NCT) to compare networks from female and male subjects in our dataset (van Borkulo et al., 2016). The NCT rearranges the samples to test whether two networks are invariant with respect to global strength (sum of all edge weights), network structure and edge values.

Network stability State-of-the-art stability analyses (Epskamp and Fried, 2018) were carried out using the same methodology used in our previous studies (Briganti et al., 2018). An edge weight difference test was performed to answer the question "is edge A significantly stronger than edge B?".

\subsubsection{Directed Acyclic Graph}

In Bayesian networks an edge may represent a causal pathway between two nodes. The structure of a Bayesian network can be estimated using constraint-based algorithms, that analyze conditional independence relations among the nodes in the network. Constraint-based algorithms generate a network model that can be interpreted as a causal model even in observational data, under assumptions that in clinical terms exclude confounding and sampling bias.

In this paper we used the PC algorithm, a constraint-based algorithm (Spirtes et al., 1993). To estimate a network model, the PC algorithm first estimates an undirected network model in which all pairs of nodes are connected, then deletes edges between conditional independent pairs of variables, and directs edges starting with v-structures (two disconnected nodes causing a third node).

The estimated network was investigated using stability analysis through bootstrapping. Afterwards, a network is reported with a minimum connection strength (\% of fitted networks in which a given connection appears) of 85 and a minimum connection direction (\% of fitted networks in which a given connection has a given direction) of 50. This resulting network therefore reports connections that are presents in more than $85 \%$ of fitted networks; moreover, these connections present a direction (for instance, from node A to node B) which is found in more than half of the fitted networks resulting from the bootstrapping procedure.

\section{Results}

\subsection{Regularized partial correlation network}

Figure 1 represents the glasso network of items from the SDS. The network is overall positively connected. The strongest connection in the network $(0.26)$ is found between items 17 ("I feel that I am useful and needed") and 18 ("My life is pretty full"). Other strong connections include edge 1-3 ("I feel downhearted and blue"; "I have crying spells or feel like it"), edge 11-12 ("My mind is as clear as it used to be"; "I find it easy to do the things I used to") and edge 16-17 ("I find it easy to make decisions"; "I feel that I am useful 


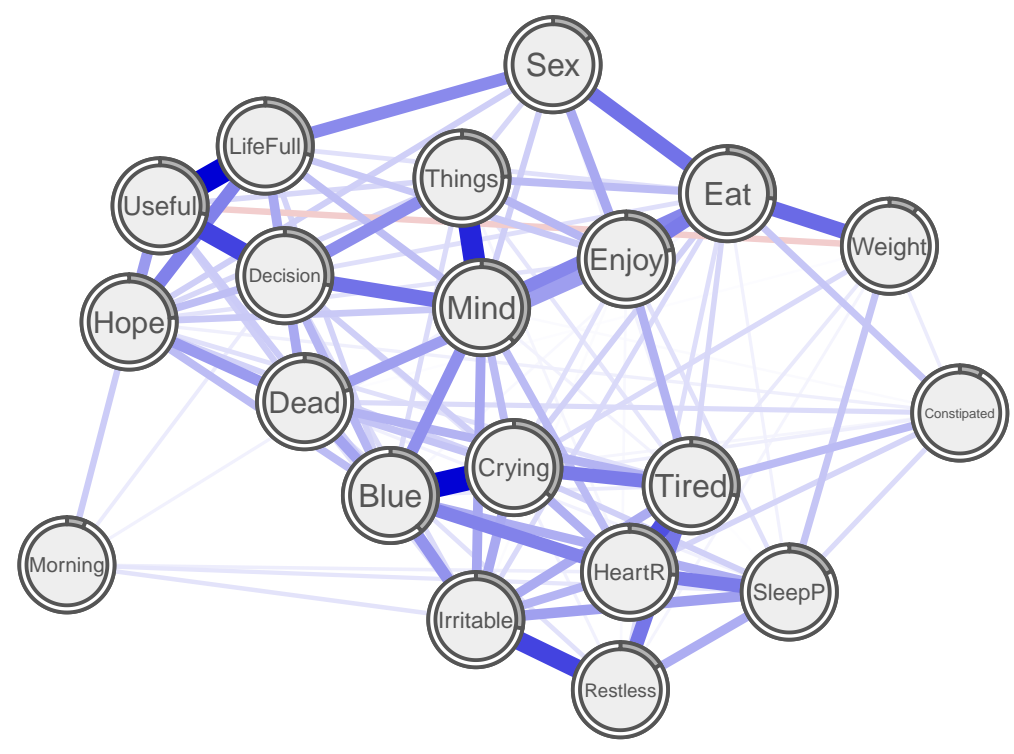

Blue: I feel down hearted and blue Morning: Morning is when I feel the best SleepP: I have trouble sleeping at night Eat: I eat as much as I used to Sex: I still enjoy sex Weight: I notice that I am losing weight Constipated: I have trouble with constipation HeartR: My heart beats faster than usual Tired: I get tired for no reason Things: I find it easy to do the things t be Restless: I am restless and cannot keep still Hope: I feel hopeful about the future Irritable: I am more irritable than usual Decision: I find it easy to make decisions Useful: I feel that l am useful and needed Deful: My ire is pretly ful er off if I were dead Enjoy: I still enjoy the things I used to do

Figure 1: Regularized partial correlation network of items from the SDS. Each node represents an item from the SDS; positive connections are blue, negative connections are red. The pie chart surrounding each node represents node predictability.

and needed"). Items 2 ("Morning is when I feel best") and 8 ("I have trouble with constipation") are poorly connected with the rest of the nodes in the network. One negative connection is found between item 7 ("I notice that I am losing weight") and 17 ("I feel that I am useful and needed").

\subsubsection{Network inference}

Mean node predictability is 0.23 , which means that on average items from the SDS share $23 \%$ of variance with surrounding items. The most predictable node is item 11 ("My mind is not as clear as it used to be"), which shares $38 \%$ of variance with surrounding items. The least predictable node is item 7 ("I notice that I am losing weight") and it shares $10 \%$ with surrounding items.

\subsubsection{Network Comparison Test}

The networks estimated from both female and male students were substantially similar and did not differ statistically regarding global strength, network structure and edge values.

\subsubsection{Network stability}

The edge weight difference test reports that the strongest edges $(17-18 ; 1-3 ; 11-12 ; 16-17 ; 13-15 ; 9-10)$ are significantly stronger than other edges in the network but are not significantly different from each other; therefore, we cannot safely interpret which edge is the strongest.

\subsection{DAG}

Figure 2 shows the DAG estimated with PC algorithm and reporting stable connections resulting from bootstrapping. The DAG shows some interesting directed connections; we will describe a few examples of such edges. Item 1 ("I feel downhearted and blue") has two outgoing edges to items 9 ("My heart beats faster than usual") and 14 ("I feel hopeful about the future"). Item 2 ("Morning is when I feel best") also has two outgoing edges to items 14 ("I feel hopeful about the future") and 19 ("I feel that others would be better off if I were dead"). Item 4 ("I have trouble sleeping at night") has three outgoing edges to items 12 


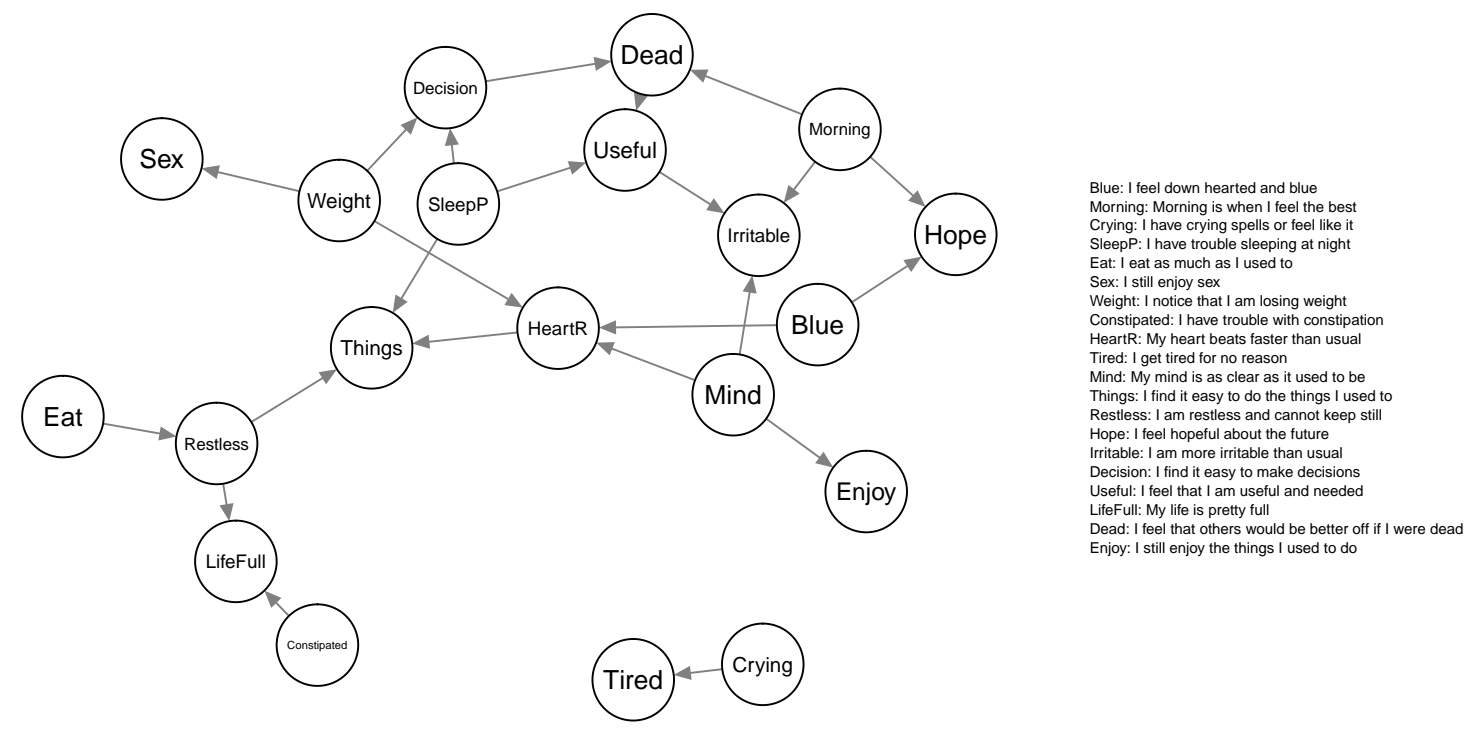

Figure 2: Directed Acyclic Graph.

("I find it easy to do the things I used to"), 16 ("I find it easy to make decisions") and 17 ("I feel that I am useful and needed"). Item 15 ("I am more irritable than usual") has three incoming connections from items 2 ("Morning is when I feel the best"), 11 ("My mind is as clear as it used to be"), and 17 ("I feel that I am useful and needed"). Item 19 ("I feel that others would be better off if I were dead") has two incoming connections from items 2 ("Morning is when I feel best") and 16 ("I find it easy to make decisions"). Regression coefficients for nodes in the DAG are positive, which means that nodes from the DAG are overall positively connected: these analyses are reported in the supplementary materials.

\section{Discussion}

This is to our knowledge the first application of network analysis to the SDS. This study, conducted in a non-clinical large sample of French-speaking university students, sheds light on the network structure of depression items, which may replicate in clinical samples. The regularized partial correlation network shows that items from the SDS are overall positively connected, but the degree of connectivity of a given node from the SDS to other nodes (also defined as node degree in network science) varies; for instance, we identified two items from the SDS (2 and 8) which are overall poorly connected to the rest of the network.

From a network point of view, disconnected items may be considered to be independent controlling for other nodes in the network. Some connections are stronger than others as reported in the stability analyses. Some strong edges however are shared between two items that might measure the same symptom, such as edge 1-3 ("I feel downhearted and blue"; "I have crying spells or feel like it"); in this case, this connection should be interpreted as shared variance between the two items (Fried and Cramer, 2017). In most cases in this network, however, connections offer novel insight as to how symptoms interact; we will detail one example to show such insight offered by our study. For instance, the strongest connection in the network between items 17 ("I feel that I am useful and needed") and 18 ("My life is pretty full") may be interpreted as such: at the level of the observed group and controlling for other nodes in the network, it is more likely not to feel useful and needed if one's life is empty, and vice-versa; if this same edge were to replicate at the individual network of a person diagnosed with depression, intervening on one's feeling of having a "full life" may alleviate the feeling of being useless, and vice-versa.

Node predictability was used to assess the connectivity of items from the SDS. Item 11 ("My mind is not as clear as it used to be") shows the highest node predictability value, which means it shares the most 
variance with surrounding nodes. As reported in the DAG structure, this item receives many incoming edges from different surrounding items; we may therefore consider item 11 as the most controllable item in the network. Item 11 may therefore not be a viable target for clinical intervention if a similar network structure were to replicate in a clinical sample, since it may be considered as the consequence of surrounding symptoms. Moreover, the connectivity estimate of the network structure presented in this study resonate with other network studies on depression symptoms: for instance, having concentration problems (one of the many interpretation of item 11) has been reported in other network studies on depression to be a highly connected item; similarly, weight changes (item 7) is known to have a poor connectivity (Fried et al., 2016). Future studies may also endeavor to translate the causal meaning of node predictability in other psychometric tools: however, it is interesting to note how the connectivity of network symptoms seem to replicate well across different samples and with different psychometric tools. The high replicability of network structures has been described before and is an important feature of the network approach to psychopathology (Fried et al., 2018): such replicability seems to extend to network structures from different tools (and across self-reported and observer measures).

The DAG offers additional insight as to how symptoms from the SDS may cause each other. We described several symptoms that are mainly causing other symptoms in the network, such as item 1 ("I feel downhearted and blue"), item 2 ("Morning is when I feel best") and item 4 ("I have trouble sleeping at night"). These symptoms might be considered as viable targets for clinical intervention if the same network structure were to replicate in clinical samples.

The two network models proposed in this paper present similarities as well as differences: for instance, both the regularized partial correlation network and the DAG contain positive edges; however, nodes that connect well in the regularized partial correlation network do not connect in the DAG such as the redundant items discussed in the results. The reason the different definitions of the two models: in a DAG two nodes are not automatically connected when they share a common child node, but they will be connected in the corresponding partial correlation network because of the indirect dependence conditional on that child node.

Combining different approaches when using networks to study mental disorders can therefore prove useful: DAGs can help put into perspective the information gathered with inference tools, such as considering a node controllable. The paths of symptom activation can also be uncovered with DAGs, and uncovering such paths can be particularly insightful in the case of self-reported measures such as the SDS, which are meant to reflect the individual's own perception of its symptoms.

Our results must be interpreted in light of several limitations. First, our data set is composed of university students, which may limit the generalization of our findings to different samples. Second, DAG structures do not involve loops: if in a three-node network a symptom A causes symptom B and a symptom C, symptom C cannot cause symptom A (the structure is therefore acyclic); however, it is plausible to consider that in the case of depression symptoms, certain symptoms may activate each other in a loop. Third, causation may be inferred from a connection between two nodes in a DAG assuming there are no confounding or sampling bias.

Further studies may endeavor to replicate our findings in different samples, both non-clinical and clinical, to replicate network structure (both Bayesian and non-Bayesian) of symptoms described in the SDS, and look for similarities as well as differences between different models of depression components.

\section{References}

American Psychiatric Association (2013). Diagnostic and Statistical Manual of Mental Disorders. American Psychiatric Association, fifth edition.

Borsboom, D. (2008). Psychometric perspectives on diagnostic systems. Journal of Clinical Psychology, 64(9):1089-1108.

Borsboom, D. (2017). A network theory of mental disorders. World Psychiatry, 16(1):5-13.

Borsboom, D. and Cramer, A. O. (2013). Network Analysis: An Integrative Approach to the Structure of Psychopathology. Annual Review of Clinical Psychology, 9(1):91-121. 
Briganti, G., Fried, E. I., and Linkowski, P. (2019). Network analysis of Contingencies of Self-Worth Scale in 680 university students. Psychiatry Research, 272:252-257.

Briganti, G., Kempenaers, C., Braun, S., Fried, E. I., and Linkowski, P. (2018). Network analysis of empathy items from the interpersonal reactivity index in 1973 young adults. Psychiatry Research, 265:87-92.

Cramer, A. O. J., Borkulo, C. D. v., Giltay, E. J., Maas, H. L. J. v. d., Kendler, K. S., Scheffer, M., and Borsboom, D. (2016). Major Depression as a Complex Dynamic System. PLOS ONE, 11(12):e0167490.

Epskamp, S., Cramer, A. O. J., Waldorp, L. J., Schmittmann, V. D., and Borsboom, D. (2012). qgraph: Network Visualizations of Relationships in Psychometric Data. Journal of Statistical Software, 48(4).

Epskamp, S. and Fried, E. I. (2018). A tutorial on regularized partial correlation networks. Psychological Methods, 23(4):617-634.

Fried, E. I., Borkulo, C. D. v., Cramer, A. O. J., Boschloo, L., Schoevers, R. A., and Borsboom, D. (2017). Mental disorders as networks of problems: a review of recent insights. Social Psychiatry and Psychiatric Epidemiology, 52(1):1-10.

Fried, E. I. and Cramer, A. O. J. (2017). Moving Forward: Challenges and Directions for Psychopathological Network Theory and Methodology. Perspectives on Psychological Science: A Journal of the Association for Psychological Science, 12(6):999-1020.

Fried, E. I., Eidhof, M. B., Palic, S., Costantini, G., Huisman-van Dijk, H. M., Bockting, C. L. H., Engelhard, I., Armour, C., Nielsen, A. B. S., and Karstoft, K.-I. (2018). Replicability and Generalizability of Posttraumatic Stress Disorder (PTSD) Networks: A Cross-Cultural Multisite Study of PTSD Symptoms in Four Trauma Patient Samples. Clinical Psychological Science.

Fried, E. I., Epskamp, S., Nesse, R. M., Tuerlinckx, F., and Borsboom, D. (2016). What are 'good' depression symptoms? comparing the centrality of dsm and non-dsm symptoms of depression in a network analysis. Journal of affective disorders, 189:314-320.

Fried, E. I. and Haslbeck, J. M. (2019). Using network analysis to examine links between individual depression symptoms, inflammatory markers, and covariates. Psychological Medicine, pages 1-9.

Fried, E. I. and Nesse, R. M. (2015). Depression is not a consistent syndrome: An investigation of unique symptom patterns in the STAR*D study. Journal of Affective Disorders, 172:96-102.

Friedman, J., Hastie, T., and Tibshirani, R. (2014). glasso: Graphical lasso- estimation of Gaussian graphical models.

Fruchterman, T. M. J. and Reingold, E. M. (1991). Graph drawing by force-directed placement. Software: Practice and Experience, 21(11):1129-1164.

Galderisi, S., Rucci, P., Kirkpatrick, B., Mucci, A., Gibertoni, D., Rocca, P., Rossi, A., Bertolino, A., Strauss, G. P., Aguglia, E., Bellomo, A., Murri, M. B., Bucci, P., Carpiniello, B., Comparelli, A., Cuomo, A., Berardis, D. D., Dell'Osso, L., Fabio, F. D., Gelao, B., Marchesi, C., Monteleone, P., Montemagni, C., Orsenigo, G., Pacitti, F., Roncone, R., Santonastaso, P., Siracusano, A., Vignapiano, A., Vita, A., Zeppegno, P., and Maj, M. (2018). Interplay Among Psychopathologic Variables, Personal Resources, Context-Related Factors, and Real-life Functioning in Individuals With Schizophrenia: A Network Analysis. JAMA Psychiatry, 75(4):396-404.

Goldberg, D. (2011). The heterogeneity of "major depression". World Psychiatry, 10(3):226-228.

Hamilton, M. (1960). A rating scale for depression. Journal of Neurology, Neurosurgery, and Psychiatry, $23: 56-62$.

Haslbeck, J. M. B. and Fried, E. I. (2017). How predictable are symptoms in psychopathological networks? A reanalysis of 18 published datasets. Psychological Medicine, 47(16):2767-2776. 
Haslbeck, J. M. B. and Waldorp, L. J. (2016). mgm: Structure Estimation for Time-Varying Mixed Graphical Models in high-dimensional Data. arXiv preprint:1510.06871v2.

Kalisch, M., Mächler, M., Colombo, D., Maathuis, M. H., and Bühlmann, P. (2012). Causal Inference Using Graphical Models with the R Package pcalg. Journal of Statistical Software, 47(1):1-26.

Lopez, A. D., Mathers, C. D., Ezzati, M., Jamison, D. T., and Murray, C. J., editors (2006). Global Burden of Disease and Risk Factors. World Bank, Washington (DC).

McNally, R. J., Mair, P., Mugno, B. L., and Riemann, B. C. (2017). Co-morbid obsessive-compulsive disorder and depression: a Bayesian network approach. Psychological Medicine, 47(7):1204-1214.

Moffa, G., Catone, G., Kuipers, J., Kuipers, E., Freeman, D., Marwaha, S., Lennox, B. R., Broome, M. R., and Bebbington, P. (2017). Using Directed Acyclic Graphs in Epidemiological Research in Psychosis: An Analysis of the Role of Bullying in Psychosis. Schizophrenia Bulletin, 43(6):1273-1279.

Mullarkey, M. C., Marchetti, I., and Beevers, C. G. (2018). Using Network Analysis to Identify Central Symptoms of Adolescent Depression. Journal of Clinical Child E3 Adolescent Psychology, 0(0):1-13.

Robinaugh, D., Haslbeck, J. M. B., Waldorp, L., Kossakowski, J. J., Fried, E. I., Millner, A., McNally, R. J., van Nes, E. H., Scheffer, M., Kendler, K. S., and Borsboom, D. (2019). Advancing the Network Theory of Mental Disorders: A Computational Model of Panic Disorder.

Scutari, M. (2010). Learning Bayesian Networks with the bnlearn R Package. Journal of Statistical Software, $35(1): 1-22$.

Scutari, M. and Denis, J.-B. (2015). Bayesian Networks: With Examples in R.

Spirtes, P., Glymour, C., and Scheines, R. (1993). Discovery Algorithms for Causally Sufficient Structures. In Spirtes, P., Glymour, C., and Scheines, R., editors, Causation, Prediction, and Search, Lecture Notes in Statistics, pages 103-162. Springer New York, New York, NY.

St Quinton, T. and Stain, H. J. (2019). A network approach to depressive disorders. Journal of RationalEmotive 83 Cognitive-Behavior Therapy, pages 1-13.

van Borkulo, C. D., Borsboom, D., Epskamp, S., Blanken, T. F., Boschloo, L., Schoevers, R. A., and Waldorp, L. J. (2014). A new method for constructing networks from binary data. Scientific Reports, 4:5918.

van Borkulo, C. D., Epskamp, S., and Millner, A. J. (2016). NetworkComparisonTest: Statistical Comparison of Two Networks Based on Three Invariance Measures.

Wichers, M. (2014). The dynamic nature of depression: a new micro-level perspective of mental disorder that meets current challenges. Psychological medicine, 44(7):1349-1360.

Zung, W. W. (1965). A self-rating depression scale. Archives of General Psychiatry, 12:63-70. 